\title{
Sagittal Alignment of a Strut Graft Affects Graft Subsidence and Clinical Outcomes of Anterior Cervical Corpectomy and Fusion
}

\author{
Koun Yamauchi ${ }^{1}$, Kazunari Fushimi ${ }^{2}$, Kei Miyamoto $^{3}$, Akira Hioki ${ }^{4}$, Katsuji Shimizu ${ }^{3}$, Haruhiko Akiyama ${ }^{2}$ \\ ${ }^{1}$ Department of Orthopedic Surgery, Akita Hospital, Chiryu, Japan \\ ${ }^{2}$ Department of Orthopedic Surgery, Gifu University School of Medicine, Gifu, Japan \\ ${ }^{3}$ Department of Orthopedic Surgery, Spine Center, Gifu Municipal Hosptial, Gifu, Japan \\ ${ }^{4}$ Department of Orthopedic Surgery, Spine Center, Matsunami General Hosptial, Gifu, Japan
}

\section{Study Design: Retrospective study.}

Purpose: The purpose of this study was to investigate the influence of sagittal alignment of the strut graft on graft subsidence and clinical outcomes after anterior cervical corpectomy and fusion (ACCF).

Overview of Literature: ACCF is a common technique for the treatment of various cervical pathologies. Although graft subsidence sometimes occurs after ACCF, it is one cause for poor clinical results. Malalignment of the strut graft is probably one of the factors associated with graft subsidence. However, to the best of our knowledge, no prior reports have demonstrated correlations between the alignment of the strut graft and clinical outcomes.

Methods: We evaluated 56 patients (33 men and 23 women; mean age, 59 years; range, 33-84 years; 45 with cervical spondylotic myelopathy and 11 with ossification of the posterior longitudinal ligament) who underwent one- or two-level ACCF with an autogenous fibular strut graft and anterior plating. The Japanese Orthopaedic Association (JOA) score recovery ratio for cervical spondylotic myelopathy was used to evaluate clinical outcomes. The JOA score and lateral radiograms were evaluated 1 week and 1 year postoperatively. Patients were divided into two groups (a straight group [group I] and an oblique group [group Z]) based on radiographic assessment of the sagittal alignment of the strut graft.

Results: Group I showed a significantly greater JOA score recovery ratio $(p<0.05)$ and a significantly lower graft subsidence than group $Z(p<0.01)$.

Conclusions: Our findings suggest that a straight alignment of the strut graft provides better clinical outcomes and lower incidence of graft subsidence after ACCF. In contrast, an oblique strut graft can lead to significantly increased strut graft subsidence and poor clinical results.

Keywords: Cervical vertebrae; Spinal cord compression; Clinical study; Spinal fusion; Bone transplantation 


\section{Introduction}

Anterior cervical corpectomy and fusion (ACCF) is commonly employed in patients with cervical spondylotic myelopathy (CSM), cervical ossification of the posterior longitudinal ligament (OPLL), and trauma [1-5]. ACCF is particularly effective for direct removal of anterior bony spurs and disc fragments, OPLL, and correction of deformities [6-9]. A recent meta-analysis showed that ACCF resulted in better clinical outcomes and shorter hospital stay than posterior surgery, such as laminoplasty [10]. In contrast, ACCF is associated with a greater incidence of serious complications than surgery performed via a posterior approach.

Chen et al. [6] reported that graft subsidence is one of the factors associated with poor clinical outcomes. Various factors have been reported as potential risk factors for graft subsidence, such as age, sex, bone mineral density (BMD), number of corpectomies, intraoperative over-distraction of the neck, and endplate preparation. However, definitive factors remain controversial [11-17]. On the other hand, postoperative sagittal alignment of the cervical spine is a factor related to clinical outcomes. Some reports demonstrated that maintenance of postoperative cervical lordosis is important for achieving a successful postoperative result $[18,19]$. However, to the best of our knowledge, no study has focused on the alignment of the strut graft after ACCF. When the number of resected vertebrae increases, the strut graft must be longer. Therefore, the alignment of the strut graft could be more significant in cases involving multilevel ACCF.

We aimed to investigate the relationship between the alignment of the strut graft and strut graft subsidence by evaluating radiological and clinical results after ACCF. In addition, we investigated other potential factors that influence clinical outcomes.

\section{Materials and Methods}

\section{Patients}

This retrospective study was conducted with the approval of the Institutional Review Board. Patients received a written and verbal explanation and then underwent oneor two-level ACCF between 2004 and 2011. A total of 56 patients were included (33 men and 23 women; mean age, $59.0 \pm 11.9$ years; range, $33-84$ years). There were 45 pa- tients with CSM and 11 patients with OPLL. All patients were followed for a minimum of 1 year postoperatively. Patients with less than 1 year of follow-up, rheumatic arthritis, dialysis-related disorders, and a history of multiple (>two) surgeries of the cervical spine were excluded from this study.

\section{Surgical technique}

Patients were positioned supine with the neck slightly extended. All patients underwent standard anterior cervical corpectomy using a high-speed air drill through a left-sided approach. The posterior longitudinal ligament was then removed via microscopic surgery to achieve complete neural decompression. Most of the subchondral bony endplate was preserved to prevent graft subsidence. Finally, an autogenous fibular strut graft was inserted under manual cervical distraction. The location of the strut graft was confirmed using intraoperative fluoroscopy. The fusion area was stabilized with an anterior cervical plate. Postoperatively, all patients were required to wear a hard cervical collar for 2-3 months until bony callus was observed by computed tomography (CT).

\section{Radiographic evaluation}

\section{1) Cervical lordosis}

We assessed a cervical plain lateral radiograph taken with the patient in the standing position preoperatively and at 1 week and 1 year postoperatively. The $\mathrm{C} 2-\mathrm{C} 7$ lateral Cobb angle, which was used as a global parameter to determine cervical sagittal alignment, was defined as an angle between the line on the inferior endplate of $\mathrm{C} 2$ and the line on the inferior endplate of C7 (Fig. 1) [7]. The segmental lateral Cobb angle was measured for the fused area between the line on the superior endplate of the cranial vertebra and the line on the inferior endplate of the caudal vertebra (Fig. 1) [7]. Surgical correction of cervical lordosis was calculated by subtracting the preoperative lateral Cobb angle from the postoperative one. Preoperative cervical lordosis was defined as non-lordosis or lordosis based on the Cobb angle (non-lordosis group, Cobb angle $\leq 0^{\circ}$ and lordosis group, Cobb angle $>0^{\circ}$ ), and patients with segmental lordosis was divided into two groups in a similar manner (non-lordosis, Cobb angle $\leq 0^{\circ}$ and lordosis, Cobb angle $>0^{\circ}$ ). Loss of correction was calculated by subtracting the lateral Cobb angle measured 1 year post- 

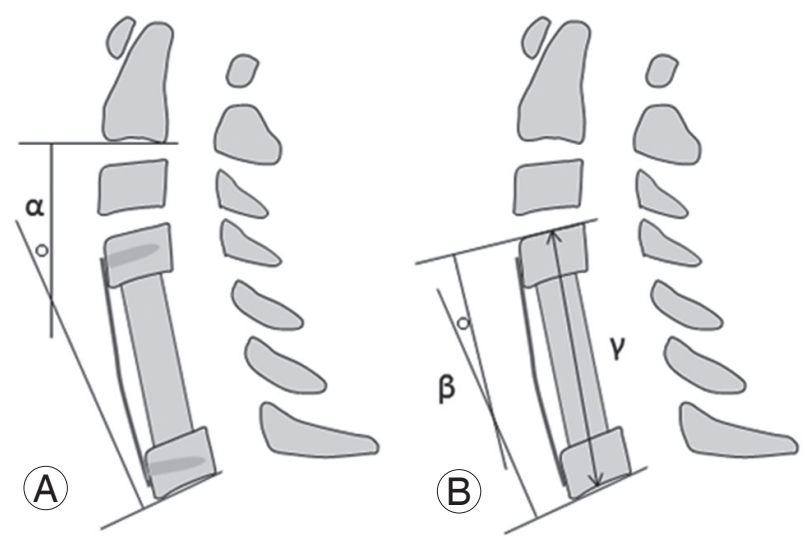

Fig. 1. Typical examples of sagittal Cobb angle and span of the fused area. The C2-C7 lateral Cobb angle ( $\alpha$ ) (A), as a global cervical sagittal alignment, and segmental lateral Cobb angle $(\beta)(B)$ were measured using Cobb's method. (B) The span of the fused area $(\gamma)$ was measured from the midpoint of the superior endplate to that of the inferior endplate.

operatively from that measured 1 week postoperatively.

\section{2) Span of the fused area}

The span of the fused area was defined as the distance between the midpoint of the superior endplate of the cranial vertebra and that of the inferior endplate of the caudal vertebra (Fig. 1) [7]. The distracted span was calculated by subtracting the preoperative intervertebral span from that measured 1 week postoperatively (shortened group, $\leq 0$ $\mathrm{mm}$; same group, $0-3 \mathrm{~mm}$; and expanded group, $>3 \mathrm{~mm}$ ). Graft subsidence was quantified by the difference between the span of the fused area measured 1 week and that of the fused area measured 1 year postoperatively (nonsubsidence group, $<3 \mathrm{~mm}$ and subsidence group, $\geq 3 \mathrm{~mm}$ ).

These radiographic evaluations were performed by a trained image analyst, who measured the $\mathrm{C} 2-\mathrm{C} 7$ lateral Cobb angle, segmental lateral Cobb angle, and span of the fused area of 20 patients two times separately. The intraclass correlation coefficient was 0.99 (95\% confidence interval [CI], 0.98-1.0) for the C2-C7 lateral Cobb angle, 0.98 (95\% CI, 0.96-0.99) for the segmental lateral Cobb angle, and 1.0 (95\% CI, 0.99-1.0) for the span of the fused area. Therefore, the reliabilities of the measurements were excellent.

\section{3) Alignment of the strut graft}

Patients were divided into two groups (groups I and Z) according to the alignment of the autogenous fibular strut graft, as determined by the standing cervical plain lateral

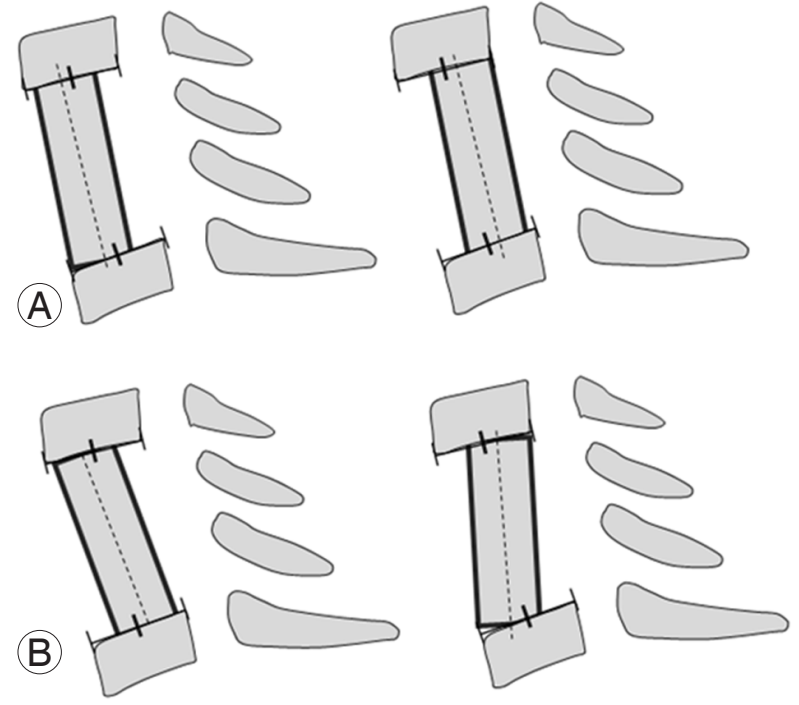

Fig. 2. Typical examples of the alignment of the strut graft. (A) The dotted line indicates the axis of the strut graft. Group I was defined as patients who received a straight strut graft, in which the strut axis was parallel to the line through the midpoints of both endplates of adjacent vertebrae. (B) Group Z was defined as patients who received an oblique strut graft, in which the strut axis was not parallel to the line through the midpoints of the endplates.

radiograph at 1 week and 1 year postoperatively. Group I included patients with a strut graft parallel to vertebrae, that is, the line through the axis of the strut graft was parallel to the line through the midpoints of both endplates of adjacent vertebrae (Fig. 2A). Group Z included patients with an oblique strut graft, that is, the line through the axis of the strut graft was not parallel to the line through the midpoints of both endplates of adjacent vertebrae (Fig. 2B).

\section{4) Fusion}

Bony fusion was evaluated by sagittal and coronal reconstructed CT. Bony fusion in CT and plain radiographs was assessed according to the method described by Hackenberg et al. [20]. Criteria for fusion were bony bridging, bony continuity between endplates, trabecular structure in the anterior strut graft, and lack of radiolucent lines around the strut graft. If three of the criteria were positive, the fusion was regarded as solid.

Radiographic evaluations with respect to the alignment of the strut graft and fusion were performed by two trained orthopedic surgeons. Intraobserver and interobserver reliability was evaluated. When intraobserver evaluations were inconsistent, the first evaluation was used. When interobserver evaluations were inconsistent, 
the evaluation performed by the first observer was used.

\section{Clinical evaluation}

Symptoms and activities of daily life were evaluated using the Japanese Orthopaedic Association (JOA) score recovery rate for CSM, which were assessed preoperatively and at 1 year postoperatively. The complete JOA score was 17 points. The JOA score recovery ratio, a normalized value postoperatively, was calculated as follows [21]. An improvement in the clinical result was defined as the JOA score recovery ratio $>0$.

Recovery ratio= $($ postoperative score-preoperative score)/(17-preoperative score)

\section{Statistical analysis}

We estimated the sample size to be 52 subjects based on a priori power analysis for Student's $t$-test as follows: type I error was 0.05 , type II error was 0.2 , and the effect size was 0.5 . Variables were classified as patient or operative factors and were statistically examined to determine whether they influenced the clinical result or subsidence. All statistical analyses were performed using SPSS for Windows ver. 12.0K (SPSS Inc., Chicago, IL, USA). In- tergroup comparisons were made using parametric tests, such as Student's $t$-test, when data were distributed normally. When data were not distributed normally, a nonparametric test was used, such as the Mann-Whitney U test and the Kruskal-Wallis test with the post hoc GamesHowell test. A $p$-value $<0.05$ was considered significant. In addition, we performed multiple logistic regression analysis using odds ratio (OR) to detect independent indicators for predicting the improvement in clinical results and occurrence of graft subsidence. All data are expressed as mean \pm standard deviation. Post hoc evaluations for effect sizes were performed using $t$ values in Student's $t$-test and $Z$ values in the Mann-Whitney $U$ test.

\section{Results}

\section{Impact of patient characteristics on clinical outcomes and graft subsidence}

There were 32 young ( $\leq 65$ years) and 24 elderly (>65 years) patients. The young group showed a significantly greater JOA score recovery ratio than the elderly group 1 year postoperatively (young group, $0.54 \pm 0.84$ and elderly group, $0.16 \pm 0.52 ; p<0.01$; medium effect size $=0.46$ ). Conversely, the patient age did not influence graft subsidence

Table 1. Impact of patients' characteristics on clinical outcomes and graft subsidence

\begin{tabular}{|c|c|c|c|c|c|}
\hline Factor & No. & Recovery JOA scores & $p$-value & Graft subsidence (mm) & $p$-value \\
\hline Disease & & & 0.46 & & 0.85 \\
\hline CSM & 45 & $0.34 \pm 0.81$ & & $4.39 \pm 4.24$ & \\
\hline OPLL & 11 & $0.58 \pm 0.37$ & & $4.52 \pm 4.99$ & \\
\hline Sex & & & 0.07 & & 0.26 \\
\hline Male & 33 & $0.47 \pm 0.84$ & & $4.81 \pm 4.49$ & \\
\hline Female & 23 & $0.27 \pm 0.59$ & & $3.83 \pm 4.12$ & \\
\hline Age & & & $<0.01$ & & 0.49 \\
\hline Young ( $\leq 65 \mathrm{yr}$ ) & 32 & $0.54 \pm 0.84^{\mathrm{al}}$ & & $4.12 \pm 4.34$ & \\
\hline Elderly (>65 yr) & 24 & $0.16 \pm 0.52^{\mathrm{al}}$ & & $4.82 \pm 4.39$ & \\
\hline C2-C7 lordosis & & & 0.19 & & 0.21 \\
\hline Non-lordosis & 23 & $0.43 \pm 0.94$ & & $4.00 \pm 4.92$ & \\
\hline Lordosis & 33 & $0.35 \pm 0.57$ & & $4.71 \pm 3.92$ & \\
\hline Segmental lordosis & & & 0.35 & & 0.56 \\
\hline Non-lordosis & 12 & $0.57 \pm 0.47$ & & $3.80 \pm 4.66$ & \\
\hline Lordosis & 44 & $0.34 \pm 0.80$ & & $4.57 \pm 4.29$ & \\
\hline
\end{tabular}

Values are presented as mean \pm standard deviation.

JOA, Japanese Orthopaedic Association; CSM, cervical spondylotic myelopathy; OPLL, ossification of the posterior longitudinal ligament. ${ }^{a)} p<0.01$. 
(young group, 4.12 \pm 4.34 and elderly group, $4.82 \pm 4.39$; $p=0.49)$. Other patient characteristics, including original diseases (CSM/OPLL) and sex (male/female), did not affect the JOA score recovery ratio and graft subsidence (Table 1).

\section{Effect of operative procedures on clinical outcomes and graft subsidence}

No significant difference was observed in the number of resected vertebrae (number of corpectomies). The JOA score recovery ratio was $0.21 \pm 1.06$ in the one-level and $0.48 \pm 0.51$ in the two-level corpectomy groups $(p=0.44)$. There also were no differences in graft subsidence between the groups $(3.78 \pm 4.10$ and $4.75 \pm 4.48$, respectively; $p=0.28$ ) (Table 2).

\section{Radiographic evaluations}

1) Cervical lordosis

Preoperative cervical lordosis (C2-C7 Cobb angle) did not correlate with clinical outcomes or graft subsidence. The JOA score recovery ratio was $0.43 \pm 0.94$ in the non-lordosis group and $0.35 \pm 0.57$ in the lordosis group $(p=0.19)$. Similarly, segmental lordosis showed no impact on the JOA score recovery ratio (non-lordosis group, $0.57 \pm 0.47$ and lordosis group, $0.34 \pm 0.80 ; p=0.35$ ) or graft subsidence ( $\mathrm{C} 2-\mathrm{C} 7$ lordosis, $p=0.21$ and segmental lordosis, $p=0.56$ ) (Table 1). Correction of segmental lordosis was observed in 30 patients, whereas $\mathrm{C} 2-\mathrm{C} 7$ lordosis was corrected in 24 patients 1 week postoperatively. The JOA score recovery ratio and graft subsidence were not significantly different between the corrected and noncorrected groups (C2-C7 lordosis: non-corrected vs. cor-

Table 2. Effect of surgical procedure and radiological parameters on clinical outcomes

\begin{tabular}{|c|c|c|c|c|c|}
\hline Parameters & No. & Recovery rate JOA scores & $p$-value & Graft subsidence (mm) & $p$-value \\
\hline No. of corpectomies & & & 0.44 & & 0.28 \\
\hline 1 & 20 & $0.21 \pm 1.06$ & & $3.78 \pm 4.10$ & \\
\hline 2 & 36 & $0.48 \pm 0.51$ & & $4.75 \pm 4.48$ & \\
\hline Correction of C2-C7 lordosis & & & 0.26 & & 0.57 \\
\hline Non-corrected & 32 & $0.27 \pm 0.90$ & & $4.41 \pm 4.06$ & \\
\hline Corrected & 24 & $0.56 \pm 0.44$ & & $4.42 \pm 4.79$ & \\
\hline Correction of segmental lordosis & & & 0.85 & & 0.86 \\
\hline Non-corrected & 26 & $0.42 \pm 0.58$ & & $3.84 \pm 3.47$ & \\
\hline Corrected & 30 & $0.38 \pm 0.88$ & & $4.89 \pm 4.95$ & \\
\hline Loss of correction; C2-7 lordosis & & & 0.41 & & 0.67 \\
\hline$>3^{\circ}$ & 20 & $0.35 \pm 0.58$ & & $4.88 \pm 4.32$ & \\
\hline$\leq 3^{\circ}$ & 36 & $0.41 \pm 0.83$ & & $4.17 \pm 4.39$ & \\
\hline Loss of segmental lordosis & & & 0.99 & & 0.024 \\
\hline$>0^{\circ}$ & 38 & $0.43 \pm 0.55$ & & $5.39 \pm 4.73^{\mathrm{a})}$ & \\
\hline$\leq 0^{\circ}$ & 18 & $0.28 \pm 1.09$ & & $2.54 \pm 2.43^{\mathrm{al}}$ & \\
\hline Length of distraction & & & 0.93 & & 0.46 \\
\hline Shortened & 19 & $0.43 \pm 0.52$ & & $3.28 \pm 3.55$ & \\
\hline Same & 20 & $0.46 \pm 0.49$ & & $7.65 \pm 4.82$ & \\
\hline Expanded & 17 & $0.29 \pm 1.14$ & & $3.86 \pm 4.19$ & \\
\hline Subsidence of the fibular strut & & & 0.032 & & \\
\hline Non-subsidence & 27 & $0.49 \pm 0.91^{\text {a) }}$ & & & \\
\hline Subsidence & 29 & $0.30 \pm 0.55^{\text {a) }}$ & & & \\
\hline
\end{tabular}

Values are presented as mean \pm standard deviation. JOA, Japanese Orthopaedic Association. ${ }^{a)} p<0.05$. 
rected groups, $p=0.26$ for the JOA score and $p=0.57$ for graft subsidence and segmental lordosis: non-corrected vs. corrected groups, $p=0.85$ for JOA score and $p=0.86$ for graft subsidence). Correction of cervical lordosis (C2-C7) was lost in 20 patients 1 year postoperatively, whereas the loss of correction was $<3^{\circ}$ in 36 patients. There was no significant differences in JOA score recovery and graft subsidence between these two groups ( $p=0.41$ for JOA score recovery and $p=0.67$ for graft subsidence). Correction of segmental lordosis was lost in 38 patients 1 year postoperatively, whereas loss of correction was $<0^{\circ}$ in 18 patients. Although there was no significant difference in JOA score recovery between these two groups, significantly greater graft subsidence was observed in the group with a loss of segmental lordosis ( $p=0.99$ for JOA score recovery and $p=0.024$ for graft subsidence, a medium effect size $=0.30$ ) (Table 2).

\section{2) Span of the fused area}

The span of the fused area was shortened in 19, unchanged in 20, and expanded in 17 patients. There was no significant difference in the JOA score recovery ratio ( $p=0.93)$ or graft subsidence $(p=0.46)$ among these three groups (Table 2). Graft subsidence was observed in $29 \mathrm{pa}$ tients 1 year postoperatively. The JOA score recovery ratio was significantly better in the non-subsidence $(0.49 \pm 0.91)$ than in the subsidence $(0.30 \pm 0.55)$ groups $(p=0.032$, a small effect size $=0.29)$ (Table 2).

\section{3) Alignment of the strut graft}

The JOA score recovery ratio 1 year postoperatively was significantly greater in group I than in group Z $(0.47 \pm 0.83$ and $0.25 \pm 0.58$, respectively; $p<0.05$, a small effect size $=0.26$ ), whereas there were no significant differences in the JOA score recovery ratio between these groups 1 week postoperatively $(0.44 \pm 0.79$ and $0.25 \pm 0.64$, respectively; $p=0.19$ ). In addition, group I showed a significantly lower incidence of subsidence than group $\mathrm{Z} 1$ year postoperatively (3.66 \pm 4.39 and $5.74 \pm 4.01$, respectively; $p<0.01$, a medium effect size $=0.35$ ) (Table 3 ). The Kappa coefficient of intraobserver agreement was 0.96 and the interobserver agreement was 0.92 . The reliability of the classification was excellent.

\section{4) Bony fusion}

Bony union was radiographically observed in all patients 1 year postoperatively. The Kappa coefficient of intraobserver and interobserver agreement was 1.0. The reliability of the diagnosis was excellent.

\section{Multiple logistic regression analysis}

Among the operative factors, the alignment of the strut graft 1 year postoperatively had a significant influence on graft subsidence and clinical result (OR, 10.83; $p<0.01$ and OR, 5.56; $p<0.05$, respectively) (Table 4 ).

\section{Discussion}

In the present study, a novel finding was that straight alignment of the strut graft provided better clinical outcomes and lower incidence of graft subsidence following ACCF. In contrast, an oblique graft may lead to increased graft subsidence and poor clinical results. Although the autogenous fibular graft is not always used because of the availability of the titanium cage and allografts, our data could indicate similar events in such patients undergoing ACCF with anterior structural support.

Table 3. Correlation between alignment of the strut graft and clinical results and graft subsidence

\begin{tabular}{|c|c|c|c|c|c|}
\hline Alignment of the strut graft & No. & Recovery rate JOA scores & $p$-value & Graft subsidence (mm) & $p$-value \\
\hline 1 wk after the surgery & & & & & 0.13 \\
\hline Type I & 40 & $0.44 \pm 0.79$ & 0.19 & $4.11 \pm 4.64$ & \\
\hline Type Z & 16 & $0.25 \pm 0.64$ & & $5.04 \pm 3.74$ & \\
\hline $1 \mathrm{yr}$ after the surgery & & & & & $<0.01$ \\
\hline Type I & 35 & $0.47 \pm 0.83^{\mathrm{a})}$ & 0.048 & $3.66 \pm 4.39^{b)}$ & \\
\hline Type Z & 21 & $0.25 \pm 0.58^{\text {a) }}$ & & $5.74 \pm 4.01^{b)}$ & \\
\hline
\end{tabular}

Values are presented as mean \pm standard deviation.

JOA, Japanese Orthopaedic Association.

${ }^{\text {a) }} p<0.05$; ${ }^{\text {b) }} p<0.01$. 
Table 4. Multiple logistic regression analysis among several factors

\begin{tabular}{|c|c|c|c|c|}
\hline \multirow{2}{*}{ Operative factor } & \multirow{2}{*}{$p$-value } & \multirow{2}{*}{ OR } & \multicolumn{2}{|c|}{$95 \% \mathrm{Cl}$ for OR } \\
\hline & & & Lower & Upper \\
\hline \multicolumn{5}{|l|}{ Occurrence of the graft subsidence } \\
\hline Number of corpectomy & 0.80 & - & - & - \\
\hline Correction of C2-C7 lordosis & 0.51 & - & - & - \\
\hline Correction of segmental lordosis & 0.47 & - & - & - \\
\hline Distraction of the vertebra & 0.34 & - & - & - \\
\hline Alignment of the grafted bone & $<0.01$ & 10.83 & 2.08 & 56.36 \\
\hline \multicolumn{5}{|l|}{ Improvement of the clinical result } \\
\hline Number of corpectomy & 0.25 & - & - & - \\
\hline Correction of C2-C7 lordosis & 0.58 & - & - & - \\
\hline Correction of segmental lordosis & 0.57 & - & - & - \\
\hline Distraction of the vertebra & 0.99 & - & - & - \\
\hline Alignment of the grafted bone & 0.042 & 5.56 & 1.05 & 31.25 \\
\hline
\end{tabular}

$\mathrm{OR}$, odds ratio; $\mathrm{Cl}$, confidence interval.

One advantage of the ACCF procedure is that distraction of the anterior column and correction of deformity are possible. Larger grafts can distract the anterior column, indirectly decompressing the neural elements through increased foraminal height and unbuckling of the ligamentum flavum [22]. However, these large grafts may influence the axial load transmitted through the adjacent vertebrae. Significantly higher compressive forces on adjacent endplates were loaded with larger grafts and distraction [23].

A difference in the compressive forces on contacting endplates between straight and oblique strut grafts is not known. Increased pressure (force per square inch) on the endplate by an oblique graft has been suggested because the sharp edges of the oblique graft make the contacting area on the endplates smaller. However, even if the strut is set straight in vertebrae, it must be kept straight until the bony union is complete. In fact, five patients with straight strut grafts immediately postoperatively were observed to have oblique alignment 1 year postoperatively. Therefore, no significant difference was observed in the JOA score recovery rate 1 year postoperatively between those with straight and oblique strut grafts 1 week postoperatively. We believed that causative factors in the alteration of the alignment of strut grafts are complicated, and we could not identify what may have altered the alignment of the strut graft in this study. Future research should investigate the changes in the alignment of the strut graft over time.
Excessive autogenous bone graft subsidence (over 3 $\mathrm{mm}$ ) was recently reported to be associated with poor clinical outcomes [6]. We found that the subsidence group showed a poorer JOA score recovery ratio than the nonsubsidence group. Then, why did the subsidence group have poorer clinical result? Subsidence could lead to slack and bulging of the ligamentum flavum, resulting in recurrence of spinal cord and nerve root impingement [6]. Some investigators reported that subsidence could cause catastrophic failure of fixation or screw breakages, thus requiring revision surgery in some cases $[8,9,17]$. In this study, two patients experienced backout of the plate and screws within 1 month postoperatively. However, proper alignment of the strut graft 1 year postoperatively was maintained in these patients, without any subsidence. The results from reoperation in these patients revealed the main cause of revision surgery to be anterior migration of the plate with screw backout due to insufficient resection of an anterior bony spur. In contrast, the alignment of the strut graft and neural recovery were good in those cases.

Other studies have shown that kyphotic deformities that accompany graft subsidence could cause further deleterious effects [19,21]. Our study showed that the correction of segmental and C2-C7 lordosis may not lead to significant subsidence and improvement of clinical results. However, we showed that the loss of segmental lordosis, not $\mathrm{C} 2-\mathrm{C} 7$ lordosis, may accompany graft subsidence. Therefore, we considered that malalignment of the strut 
graft may induce graft subsidence, which may lead to the loss of segmental lordosis (not to extent of loss of C2-C7 lordosis).

Then, which other factors induced graft subsidence? $\mathrm{Pa}-$ tient age is reported to be a risk factor for graft subsidence because of low BMD, in other words, a fragile vertebral body and bony endplates are potential risk factors [10]. However, patient age was not found to be a risk factor for graft subsidence in the present study, but was an influential factor for neurological recovery. We suggested that younger patients had a higher potential for neural recovery after spinal cord decompression than elderly patients.

There are some limitations in this study. Although poor bone quality in the vertebra and end plate thickness should affect graft subsidence, BMD was not evaluated. However, age or sex may provide an indirect measure of BMD. In addition, there were limitations in the instrumental factors. Two types of dynamic anterior cervical plates were used in this study based on the mechanism: one as translation through the plate and the other as translation through the screw holes. We did not evaluate the differences in outcome between the types of implants. The possible impact of these differences in instruments should be evaluated in further studies.

\section{Conclusions}

We concluded that alignment of the strut graft significantly affected clinical outcomes and subsidence. Our results suggested that straight insertion of the strut graft in alignment with the line through adjacent vertebra and maintenance of a straight alignment until achieving bony union are important for supporting positive clinical outcomes.

\section{Conflict of Interest}

No potential conflict of interest relevant to this article was reported.

\section{References}

1. Rao RD, Gourab K, David KS. Operative treatment of cervical spondylotic myelopathy. J Bone Joint Surg Am 2006;88:1619-40.

2. Witwer BP, Trost GR. Cervical spondylosis: ventral or dorsal surgery. Neurosurgery 2007;60:S130-6.

3. Yonenobu K, Fuji T, Ono K, Okada K, Yamamoto T,
Harada N. Choice of surgical treatment for multisegmental cervical spondylotic myelopathy. Spine (Phila Pa 1976) 1985;10:710-6.

4. Kawakami M, Tamaki T, Iwasaki H, Yoshida M, Ando M, Yamada H. A comparative study of surgical approaches for cervical compressive myelopathy. Clin Orthop Relat Res 2000;(381):129-36.

5. Fessler RG, Steck JC, Giovanini MA. Anterior cervical corpectomy for cervical spondylotic myelopathy. Neurosurgery 1998;43:257-65.

6. Chen Y, Chen D, Guo Y, et al. Subsidence of titanium mesh cage: a study based on 300 cases. J Spinal Disord Tech 2008;21:489-92.

7. Oh MC, Zhang HY, Park JY, Kim KS. Two-level anterior cervical discectomy versus one-level corpectomy in cervical spondylotic myelopathy. Spine (Phila Pa 1976) 2009;34:692-6.

8. Kanayama M, Hashimoto T, Shigenobu K, Oha F, Ishida T, Yamane S. Pitfalls of anterior cervical fusion using titanium mesh and local autograft. J Spinal Disord Tech 2003;16:513-8.

9. van Jonbergen HP, Spruit M, Anderson PG, Pavlov PW. Anterior cervical interbody fusion with a titanium box cage: early radiological assessment of fusion and subsidence. Spine J 2005;5:645-9.

10. Sun Y, Li L, Zhao J, Gu R. Comparison between anterior approaches and posterior approaches for the treatment of multilevel cervical spondylotic myelopathy: a meta-analysis. Clin Neurol Neurosurg 2015;134:28-36.

11. Hasegawa K, Abe M, Washio T, Hara T. An experimental study on the interface strength between titanium mesh cage and vertebra in reference to vertebral bone mineral density. Spine (Phila Pa 1976) 2001;26:957-63.

12. Dorai Z, Morgan H, Coimbra C. Titanium cage reconstruction after cervical corpectomy. J Neurosurg 2003;99:3-7.

13. Narotam PK, Pauley SM, McGinn GJ. Titanium mesh cages for cervical spine stabilization after corpectomy: a clinical and radiological study. J Neurosurg 2003;99:172-80.

14. Hee HT, Majd ME, Holt RT, Whitecloud TS, 3rd, Pienkowski D. Complications of multilevel cervical corpectomies and reconstruction with titanium cages and anterior plating. J Spinal Disord Tech 2003;16:18 . 
15. Lim $\mathrm{TH}$, Kwon $\mathrm{H}$, Jeon $\mathrm{CH}$, et al. Effect of endplate conditions and bone mineral density on the compressive strength of the graft-endplate interface in anterior cervical spine fusion. Spine (Phila Pa 1976) 2001;26:951-6.

16. Daubs MD. Early failures following cervical corpectomy reconstruction with titanium mesh cages and anterior plating. Spine (Phila Pa 1976) 2005;30:14026.

17. Truumees E, Demetropoulos CK, Yang KH, Herkowitz HN. Effects of disc height and distractive forces on graft compression in an anterior cervical corpectomy model. Spine (Phila Pa 1976) 2008;33:1438-41.

18. Nakase H, Park YS, Kimura H, Sakaki T, Morimoto T. Complications and long-term follow-up results in titanium mesh cage reconstruction after cervical corpectomy. J Spinal Disord Tech 2006;19:353-7.

19. Das K, Couldwell WT, Sava G, Taddonio RF. Use of cylindrical titanium mesh and locking plates in anterior cervical fusion. Technical note. J Neurosurg 2001;94:174-8.

20. Hackenberg L, Halm H, Bullmann V, Vieth V, Schneider M, Liljenqvist $U$. Transforaminal lumbar interbody fusion: a safe technique with satisfactory three to five year results. Eur Spine J 2005;14:551-8.

21. Guo Q, Bi X, Ni B, et al. Outcomes of three anterior decompression and fusion techniques in the treatment of three-level cervical spondylosis. Eur Spine J 2011;20:1539-44.

22. An HS, Evanich CJ, Nowicki BH, Haughton VM. Ideal thickness of Smith-Robinson graft for anterior cervical fusion: a cadaveric study with computed tomographic correlation. Spine (Phila Pa 1976) 1993;18:2043-7.

23. Truumees E, Demetropoulos CK, Yang KH, Herkowitz HN. Effects of disc height and distractive forces on graft compression in an anterior cervical corpectomy model. Spine (Phila Pa 1976) 2008;33:1438-41. 